\title{
Diagnóstico de la hidrocefalia de presión normal
} Diagnostic of normal pressure hydrocephalus

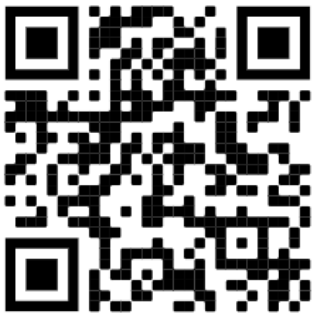

Recibido $02 / 01 / 2021$

\author{
${ }^{1}$ Dra. Priscilla Montoya Alan \\ Investigadora independiente, San José, Costa Rica \\ (D) https://orcid.org/0000-0002-0216-8961 \\ ${ }^{2}$ Dra. Katherine Murillo Alvarado \\ Investigadora independiente, San José, Costa Rica \\ https://orcid.org/0000-0002-7156-4554
}

$\begin{array}{cc}\text { Corregido } & \text { Aceptado } \\ 20 / 01 / 2021 & 02 / 02 / 2021\end{array}$

\section{RESUMEN}

La hidrocefalia normotensa es una patología infrecuente y reversible, de etiología desconocida ya que las teorías presentadas no explican la sintomatología en su totalidad. Se clasifica como idiopática cuando se presenta el cuadro clínico usual de pacientes mayores de 70 años junto con la triada de Hakim clásica y las secundarias a alguna patología cerebral que se puede presentar a cualquier edad. El diagnóstico se basa en la clínica, medida de la presión media del líquido cefalorraquídeo y ventriculomegalia en imágenes radiológicas. Presenta una correlación con la enfermedad de Alzheimer y que no son excluyentes entre sí. Entre los diagnósticos diferenciales se encuentran las demencias e incontinencias urinarias usuales en edades avanzadas. La colocación de la válvula ventrículo peritoneal sigue siendo el tratamiento de primera línea.

PALABRAS CLAVE: hidrocefalia; marcha; presión de líquido cefalorraquídeo; demencia.

\section{ABSTRACT}

Normal pressure hydrocephalus is an uncommon and reversible pathology of unknown etiology since there is no theory that could fully explain the symptomatology. It is classified as idiopathic, the usual clinical presentation is with patients older than 70 years old along with Hakim's triad and the secondary to a cerebral pathology that can happen at any age. The diagnosis is based on the clinical presentation, the measure of the cerebrospinal fluid pressure and ventriculomegaly in radiological images. It showcases a correlation with Alzheimer's disease and they do not exclude each other. In the differential diagnosis it's found dementias and urinary incontinence that are usual in advanced ages. The ventriculoperitoneal valve drain is still the first line of treatment.

KEYWORDS: hydrocephalus; gait; cerebrospinal fluid pressure; dementia.

\footnotetext{
${ }^{1}$ Médica general, graduada de la Universidad de Ciencias Médicas (UCIMED), cod. MED13645, correo: Priella86@gmail.com

2 Médica general, graduada de la Universidad de Ciencias Médicas (UCIMED), cod. MED12617, correo: Kat.murilloalvarado@gmail.com
} 


\section{INTRODUCCIÓN}

Aproximadamente un $80 \%$ del líquido cefalorraquídeo (LCR) se produce en el plexo coroideo ubicado en los ventrículos, mientras que el $20 \%$ restante se produce en el epéndimo y sector intersticial de donde se comunica hacia toda la circulación por el sistema ventricular $(1,2)$.

El término hidrocefalia deriva de las palabras en latín hidro, que significa agua y céfalo que significa cabeza. Esta se define como una dilatación activa del sistema ventricular debido a una inadecuada circulación de líquido cefalorraquídeo (LCR) desde su punto de producción y absorción (2). Usualmente cursa con aumento de la presión intracraneal, siendo ésta normalmente menos de $13 \mathrm{~mm} \mathrm{Hg} \mathrm{o} 180 \mathrm{~mm}$ $\mathrm{H} 20$ ()ㅜ).

La hidrocefalia puede producirse por el aumento en la producción del LCR, obstrucción de la circulación (por ejemplo, masas o tumor) y disminución de la reabsorción (2). La hidrocefalia puede clasificarse por su estado funcional como comunicante y no comunicante o clínica por ser aguda o crónica (4).

La hidrocefalia de presión normal idiopática (HPNi) fue descrita por Salomón Hakim, quién publicó la descripción clínica de una serie de tres pacientes que presentaban demencia progresiva, alteraciones de la marcha y dilatación variable del sistema ventricular que se acompañaba de presiones normales del LCR, por lo que se clasifica como comunicante crónica. Este tipo de HPNi ocasiona alrededor del $0.5 \%$ de las demencias y es reversible. Clasificada como idiopática cuadro en adultos mayores de 70 años junto con la triada clínica de Hakim y la secundaria a una patología (므그).

El diagnóstico se realiza mediante la clínica, características fisiológicas e imagenes y debe diferenciarse de otras demencias como la enfermedad del Alzheimer (EA) así como de otras causas de hidrocefalia. El tratamiento y pronóstico recaen sobre el diagnóstico especifico.

El objetivo de este artículo es realizar una revisión de tema sobre la hidrocefalia normotensa idiopática debido a que esta puede pasar inadvertida pues su sintomatología comparte características con otras patologías, sin embargo, su importancia radica en que esta patología puede ser reversible. Debido a que el diagnóstico es de exclusión e inespecífico, el diagnóstico será el principal enfoque de esta revisión, analizando bibliografía más actualizada para describir los criterios clínicos, hallazgos en la punción lumbar y por imágenes; así como su tratamiento el cual podría tener el potencial de cambiar el curso y la calidad de vida de quién la padece.

\section{MÉTODO}

Para este artículo se investigó y repasó el tema de hidrocefalia con sus generalidades, y se seleccionaron quince citas bibliográficas entre los años 2016-2021, se abarcan artículos de ScieLo, revistas digitales como Plos One, Biomédica, Revista española de Geriatría y Gerontología, libros de texto de Neurología como lo es el Zarranz, con la finalidad de exponer el objetivo de la manera más actualizada. Se realizó la búsqueda de los temas: "hidrocefalia", "hidrocefalia de presión normal", "fisiología del LCR" y "fisiopatología de la hidrocefalia normotensiva".

Se recolectó la información sobre epidemiología, cuadro clínico, fisiología y las teorías fisiopatológicas, diagnóstico y tratamiento del cuadro, además de su relación con el Alzheimer. 


\section{GENERALIDADES DE HIDROCEFALIA}

Aproximadamente un $80 \%$ del líquido cefalorraquídeo (LCR) se produce en el plexo coroideo ubicado en los ventrículos, mientras que el $20 \%$ restante se produce en el epéndimo y sector intersticial. Los ventrículos laterales se comunican al tercer ventrículo por medio del foramen de Monroe, $\mathrm{y}$ este se comunica al cuarto ventrículo a través del acueducto de Silvio. EL LCR abandona el sistema ventricular por el foramen de Luschka (lateral) y Magendie (medial) hacia el espacio subaracnoideo alrededor del cerebro y médula espinal. Finalmente, es reabsorbido por las granulaciones aracnoideas hacia los senos venosos y al torrente sanguíneo. La reabsorción es un evento pasivo que depende de los gradientes de presión que se ejercen entre los sectores subaracnoideo y venosos $(1,2)$.

Las hidrocefalias son una dilatación activa del sistema ventricular, ocasionada por aumento en la producción del LCR, obstrucción de la circulación y disminución de la reabsorción, que altera la dinámica normal del LCR (2).

La producción del LCR tiene una velocidad de $0.3-0.6 \mathrm{ml} / \mathrm{min}$, alrededor de $500 \mathrm{ml} / 24$ horas (1). El volumen normal de $150 \mathrm{ml}$, donde $120 \mathrm{ml}$ y $30 \mathrm{ml}$ se localizan en el espacio subaracnoideo cerebral y espinal, respectivamente (1). La presión normal del LCR es de menos de $13 \mathrm{~mm} \mathrm{Hg}$ o $180 \mathrm{~mm}$ H20 (므). Se clasifican a nivel funcional y clínica:

La clasificación funcional se subdivide en $(2,4)$ :

- Hidrocefalia no comunicante: son obstructivas como por ejemplo, los tumores, quistes u otras lesiones que físicamente obstruyen a la salida del líquido en el sistema ventricular.

- Hidrocefalia comunicante: causada por la disminución en la reabsorción, obstrucción del flujo o en casos muy raros exceso de la producciónn. Dónde pertenece la hidrocefalia normotensa.

La clasificación clínica se basa en el tiempo de evolución (4):

- Aguda

- Excepcional en personas de avanzada edad

- Se da un incremento en la presión intracraneal.

- Hidrocefalia activa cuando hay dilatación progresiva de los ventrículos con síntomas y signos de hipertensión intracraneana, como papiledema, vómitos, alteración patrón respiratorio.

- Alcanza un agrandamiento ventricular del $80 \%$ en 6 horas.

\section{- Crónica}

- Mayoritariamente en adultos.

- Balance en la producción como en la reabsorción, así se equilibran y disminuye la presión intracraneal, como lo es en el caso de la HPNi.

\section{HIDROCEFALIA NORMOTENSIVA IDIOPÁTICA}

\section{Epidemiología}

Es una enfermedad que se caracteriza por ser infrecuente 21.9 por cada 100,000 individuos, insidiosa y del adulto mayor $>70$ 
años $(\underline{6}, \underline{7})$. Su prevalencia es de $0.2 \%$ entre edades 70 a 79 años y $5.9 \%$ sobre los 80 años alrededor de $10 \%$ de los pacientes con demencia sufren de hidrocefalia de presión normal idiopática (ㅇ) $)$ No hay diferencia entre sexos, se ha documento que en casos inmediatamente posterior a la cirugía el pronostico ha mejorado entre un 24 a $80 \%$ y $50 \%$ a unos 3 años posteriores (ㅁ).

\section{Patogenia}

Entre las teorías presentadas son (4):

- Teoría clásica: la producción del LCR es mayor que su absorción. Así como se describe posible absorción insuficiente en las granulaciones aracnoideas. Por lo que, los procesos que afectan el espacio subaracnoideo ya sea hemorragias, infecciosos, trauma predisponen a la hidrocefalia.

- Teoría de expansión: las presiones iniciales de la HPNi son altas. Sin embargo, se normalizan al aumentar el tamaño ventricular.

- Teoría de la presión intracraneal fluctuante: hay aumentos intermitentes críticos de la presión intraventricular.

- Teoría del gradiente: el gradiente transependimario (diferencia entre los ventrículos y espacio subaracnoideo), es la que va a determinar la expansión de los ventrículos y no las presiones intraventriculares absolutas (4). Se describe que la expansión ventricular es posible, aún con un gradiente transependimario muy bajo. Ya que, con independencia del mismo daña la sustancia blanca periventricular y la corona radiada, incluías las proyecciones motoras sacras (lo que explicaría la triada clínica).

- Teoría del sistema glinfático y su asociación con la EA: el sistema glinfático una vía de limpieza de metabolitos cerebrales, depende del transporte de líquido conectivo a través de los espacios intersticiales, mediante los canales de aquaporina-4 en los astrocitos.

La falla en el transporte resulta en la acumulación de los desechos como Se ha valorado su relación, como la $\beta$-amiloide y proteínas tau (implicadas en desordenes neurodegenerativos) (모의). En el estudio realizado por Y.J. Bae et al. Se denota aún con sus limitantes en el estudio, que la disfunción de este sistema sí estaba presente en los pacientes con HPNi en comparación a los pacientes sin enfermedad, junto con la revisión de B.C. Reeves et al., aún se tiene el poco conocimiento sobre esta fisiopatología, que podría ser clave para futuros tratamientos. Sin embargo, es mucha la posibilidad de que esta vía sea la afectada en estos casos $(\underline{8}, \underline{9})$.

\section{Clasificación}

La hidrocefalia normotensiva se clasifica en:

- Idiopáticas: es el $50 \%$ de los casos, se desconoce su etiología. Presenta el cuadro en pacientes mayores de 70 años y con la triada clásica ( $\underline{6})$.

- Secundarias: es infrecuente, puede ocurrir a cualquier edad y la etiología es conocida. No se ha logrado determinar el tiempo de inicio de los síntomas, estos pueden ser (ㅁ):

- Hemorragia subaracnoidea: es la causa más frecuente, 
aproximadamente el $46,5 \%$ de los casos. Se ha teorizado que es ocasionada por fibrosis de las granulaciones aracnoideas resultando, menor reabsorción del LCR.

- Trauma cráneo encefálico: es el $29 \%$ de los casos y es causada secundaria al trauma que ultimadamente afecta la reabsorción del LCR.

- Malignidad (tumor cerebral) y cirugías de resección $(6,2 \%)$.

- Meningitis: tanto en casos de malignidad como inflamatorias se describe que aumentan la viscosidad del LCR por proteínas y otras sustancias, lo que termina en la dificultad de reabsorción del LCR.

- En menor frecuencia: hemorragia cerebral, enfermedad de Paget, accidentes cerebrovasculares, estenosis del acueducto $y$ radiocirugía.

En ambos casos su clínica y hallazgos diagnósticos giran en el mismo eje, por lo que no se deben tratar como identidades aparte (ㅁ). Sin embargo, se ha observado que una causa que dificulte de alguna manera la reabsorción del LCR y de manera crónica pueda ocasionar hidrocefalia normotensiva secundariamente.

\section{Diagnostico}

No hay un consenso específico, el diagnóstico se realiza entre el cuadro clínico, la punción lumbar y los hallazgos en las neuroimágenes.

a) Cuadro clínico: el motivo de consulta usual es alteración de movilidad y caídas, seguido en frecuencia por el deterioro cognitivo y síntomas urinarios (10). La triada clásica se presenta en un $50 \%$ de los casos (2), y estas son:

1. Alteraciones de la marcha (10-12):

- No hay un patrón típico

- Marcha atáxica, bradiquinética, adherido al suelo, magnético, parkinsonismo, de pasos cortos y titubeantes.

- Los problemas pueden surgir con dificultad para ascender o descender escaleras, levantarse de las sillas.

- Debilidad en miembros inferiores junto con fatiga al caminar.

2. Manifestación cognitiva y comportamental $(\underline{11}, 12)$ :

- Alteración función mental: inicio insidioso, casi patognomónica de alteración inicial de memoria reciente.

- Inicialmente sugestivos de proceso demencia subcortical: bradipsiquia, inatención, apatía. Y problemas de evocación.

- Afasia verdadera es inusual, aunque el diálogo puede estar comprometido por problemas ejecutivos o motivaciones.

- Alteración visuoespacial y visuocontructivas: la enfermedad de Alzheimer es una comorbilidad en las $\mathrm{HPNi}$, se evidencia que entre un $18-42 \%$, se sobrepone a la clínica de la HNPi (ㅁ) .

3. Incontinencia y urgencia urinaria $(\underline{3}, \underline{10}, \underline{11})$ : 
- Aparecen en etapa tardía.

- Inicialmente necesidad urgencia e incontinencia.

- Etapas avanzadas, se observa "incontinencia del lóbulo frontal", en donde la continencia es indiferente.

- mportante valorar causas adicionales de disfunción urinaria como prostatismo, estrecheces uretrales, usos diuréticos, inestabilidad del detrusor y debilidades del piso pélvico.

b) Punción lumbar: la punción lumbar revela una presión media de apertura del líquido cefalorraquídeo en niveles de variación normal ( $<180 \mathrm{~mm} \mathrm{H} 2 \mathrm{O}$ o $13 \mathrm{~mm}$ $\mathrm{Hg}$ con el paciente en la posición de cubito lateral). Actualmente se han desarrollado varias pruebas para identificar anomalías en el flujo del LCR, la más utilizada es la prueba de TAP o punción lumbar de alto flujo, donde Adam et al. (ㅁ) observaron que al drenar $50 \mathrm{ml}$ de LCR la mayoría de los pacientes presentan mejoría de sus síntomas, principalmente los relacionados al campo motor.

c) Neuroimágenes: son de suma importancia para realizar el diagnóstico y se utiliza la tomografía axial computarizada (TAC) o resonancia magnética (RM) para observar la ventriculomegalia. Las características que se observan en la ventriculomegalia incluyen $(\underline{6}, 13)$ :

- Agrandamiento mayormente de los cuernos temporales, en menor medida frontales, occipitales $y$ posteriores (FIGURA1).
- Surcos normales o aplanados.

- Índice de Evans (la relación entre los cuernos frontales y el diámetro biparietal mayor) superior a 0,3.

La RM ha sido la prueba de imagen de elección en la hidrocefalia ya que tiene una resolución mayor y se puede estudiar el flujo del LCR en tres planos diferentes (frontal, sagital y axial), lo cual es útil para diagnosticar la causa exacta de la hidrocefalia y el lugar de la obstrucción (14), con la RM, se pueden observar otros signos característicos, aunque no diagnósticos de la $\mathrm{HPNi}$; como es el aumento del vacío de flujo en el tercer ventrículo, que se produce por un aumento de la velocidad del LCR y se puede ver como una marcada hipo intensidad de señal en las secuencias T2 (14).

\section{DIAGNOSTICO DIFERENCIAL}

Usualmente la clínica de la HPNi no presenta síntomas específicos por lo que se deben pensar en otros diagnósticos. Se debe de descartar otros tipos de demencia, siendo la enfermedad de Alzheimer la más importante; ya que la ventriculomegalia es causada por la atrofia cortical, mientras que en la HPNi no se evidencia atrofia cortical (13), además que la demencia en el Alzheimer afecta principalmente la memoria reciente (ㅁ).

Otras demencias que se deben de descartar es la demencia por cuerpos de Lewy, demencia vascular (ㅁ).

Cuando se presentan datos de ataxia se debe descartar un evento cerebrovascular, masa compresiva, hemorragia subaracnoidea, entre otros (15). En cuanto a los síntomas de incontinencia urinaria, se debe descartar problemas de esfínter, infección urinaria, prostatitis (15). 
FIGURA 1. Características ventriculares

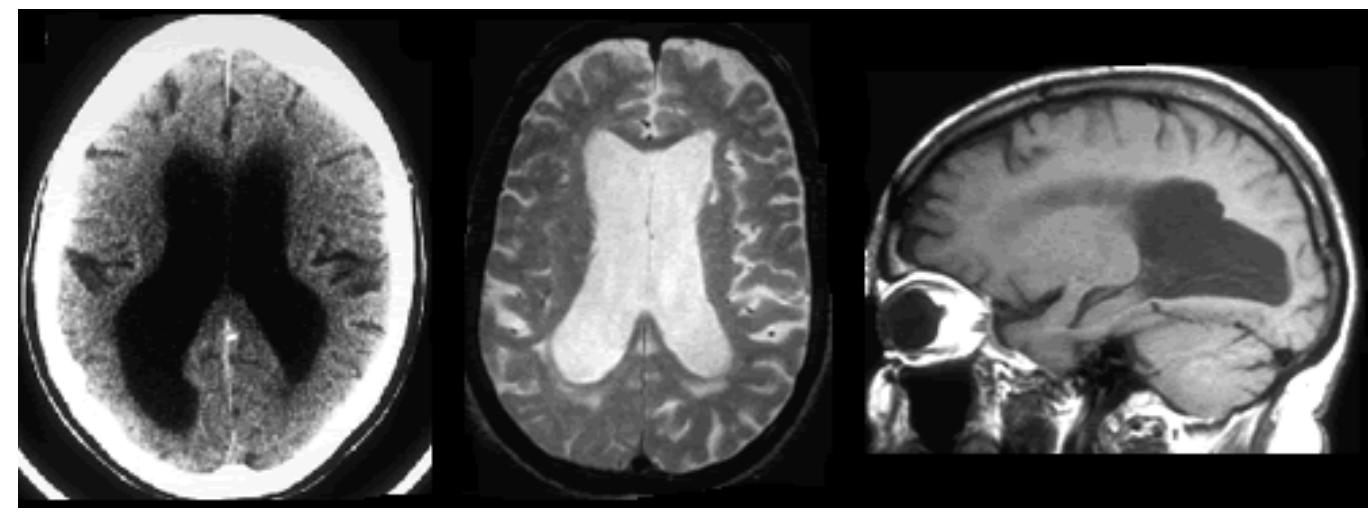

Nota: Tanto en el TAC como en la RM se observa dilatación de los ventrículos posteriores y en la segunda y tercera imagen se observa aplanamiento de los surcos cerebrales.

Fuente: Imagen de dominio público, toma de hipocampo.org https://www.hipocampo.org/galeria/galeriahidrocefNT.asp

\section{TRATAMIENTO}

El tratamiento médico no es de gran utilidad en la hidrocefalia y se utiliza como una medida temporal y junto con el tratamiento quirúrgico. La acetazolamida se ha utilizado con frecuencia, se ha observado que reduce la producción de LCR, pero sus efectos beneficiosos son mínimos y se precisan dosis altas de este fármaco, que produce acidosis metabólica, para conseguir algún efecto (14).

La instalación de una válvula derivativa de LCR representa el tratamiento definitivo para esta patología (10) y dicho procedimiento funciona en aproximadamente el $70 \%$ de los casos (16).

La cirugía para la hidrocefalia consiste en derivar LCR acumulado mediante sondas con válvulas desde un compartimiento intracraneal a otro compartimiento distal para que ese LCR se absorba por el torrente sanguíneo (14). Hay dos tipos de derivaciones, la derivación ventrículoperitoneal y la derivación ventrículo-atrial (no está indicada en la población adulta mayor por los factores cardiovasculares que puedan padecer) (17).
La derivación peritoneal actualmente es la preferida, solo está contraindicada en pacientes con espondilosis y presenta una menor tasa de infección; además la derivación ventrículo-atrial está contraindicada en la población adulta mayor que presentan factores de riesgo cardiovasculares (17). Dentro del tipo de válvulas que se utilizan en la derivación existen 4 tipos (17):

1. Válvulas programables: la baja compliance del cerebro de estos pacientes hace que sean necesarios varios ajustes de presión, este tipo de válvula al poder reajustarse externamente hace que no sea necesario una reintervención para el paciente lo que supone una ventaja y minimizar el riesgo de complicaciones postoperatorias.

Estas válvulas son las más efectivas y las que presentan menos complicaciones.

2. Válvulas de presión diferencial: incluyen las de baja presión (5-50 mm 20 ) y las de media (51-110 $\mathrm{mmH} 2 \mathrm{O})$ entre las 
más usadas. Las de baja presión presentan más mejoría clínica de la demencia y la marcha, pero las preferidas son las de media-alta presión porque presentan un riesgo de sobredrenaje bajo.

3. Válvulas gravitacionales: previene sobre drenaje en bipedestación.

4. Válvulas de flujo: no presentan diferencias en términos de mejoría, infección, fallo funcional y complicaciones respecto válvulas de presión. No son elegidas una primera opción.

5. Sistema antisifonaje: puede ser agregado a cualquier tipo de válvula, pero es importante remarcar que cuando se añade a una válvula programable desciende la incidencia de hematoma subdural al $3 \%$ y asciende la tasa de mal función a un $6 \%$.

Aunque las derivaciones han sido el pilar del tratamiento durante varias décadas, las técnicas endoscópicas son ahora más populares. Entre estas técnicas está la ventriculostomía endoscópica del tercer ventrículo, la acueductoplastia endoscópica y la colocación endoscópica de una endoprótesis en el acueducto (14).

Entre las complicaciones que pueden ocurrir con este procedimiento quirúrgico se encuentran: Mal funcionamiento de la derivación, infecciones, drenaje excesivo, lesiones cerebrales, convulsiones y las complicaciones distales como migración o desconexión de la derivación (14).

\section{CONCLUSIÓN}

La hidrocefalia normotensiva es una demencia reversible, perteneciente a las hidrocefalias comunicantes y crónicas. Puede verse en pacientes jóvenes por causas secundarias y la más común la idiopática en el adulto mayor, que es insidiosa.

Es infrecuente y se desconoce la patogenia la cual con la teoría de la sobreproducción de LCR y disminución de la absorción de este. También, se encuentra en estudio la acumulación del sistema glinfático, sin embargo, ya se logra visualizar más respuestas del por qué se correlaciona con mayor frecuencia a la enfermedad de Alzheimer.

Se concluye que en la actualidad, basado en la bibliografía seleccionada el diagnóstico es de exclusión se basa en el cuadro clínico donde la triada clásica se encuentra en un $50 \%$ de los casos, en las imágenes debe visualizarse ventriculomegalia con la RM como estudio radiológico de elección, con una presión de salida normal (menor de 180 cm de H20) y como acción terapéutica además de la colocación de la válvula es el liberar al menos $50 \mathrm{ml}$ durante esta punción lumbar, donde mejora los síntomas.

Es importante, al llegar al diagnóstico que principalmente es de exclusión otras patologías presentes durante la etapa de adulto mayor, así como con otras demencias.

\section{REFERENCIAS}

1. Vasquez H, Durango-Espinosa Y, Garcia-Ballestas E, Murlimanju B, Fernandes Joaquim A, Moscote LR, Agrawal A. Cerebrospinal fluid dynamics with its surgical implications. London Academic Publishing. [Internet] 2020;(3):459-461.https://doi.org/10.33962/roneuro-2020-075 
2. Zarranz J. Neurología. 6. ${ }^{a}$ ed. Elsevier; 2018.

3. Rodríguez Roque María Octavina, Herrera Alonso Didiesdle, Sánchez Lozano Ada, Herrera Rodríguez Marines. Hidrocefalia normotensa. Presentación de un caso. Medisur [Internet]. 2017 feb [citado 2021 Ene 03] ; 15( 1 ): 113-119. Disponible en: http://scielo.sld.cu/ scielo.php? script=sci arttext\&pid=S1727897X2017000100015\&lng=es.

4. Westover MB, DeCross E, Awad K, Bianchi M. Neurología de bolsillo. 2. ${ }^{a}$ ed. Wolters Kluwer; 2017.

5. Yasar S, Torres IJ, Lu J, Robinson J, Patel MA, Crain B, Carson KA, Hoffberger J, Batra S, Sankey E, Moghekar A, Rigamonti D. Alzhieimer's dissease pathology and shunt surgery outcome in normal pressure hydrocephalus. Plos One [Internet]. 2017 [citado 8 diciembre 2020];(12):1-11. https://doi.org/10.1371/journal.pone.0182288

6. Oliveira Louise Makarem, Nitrini Ricardo, Román Gustavo C.. Normal-pressure hydrocephalus: A critical review. Dement. neuropsychol. [Internet]. 2019 June [cited 2021 Feb 11] ; 13( 2 ): 133-143. Available from: http://www.scielo.br/scielo.php?script=sci_arttext\&pid=S1980-57642019000200133\&lng=en. Epub June 18, 2019. https://doi.org/10.1590/1980-57642018dn13-020001.

7. 7. PEDRO Matheus Kahakura Franco, SILVA Joseph Franklin Chenisz da, ROCHA Samanta Fabricio Blattes da, GERMINIANI Francisco Manoel Branco, RAMINA Ricardo, TEIVE Hélio Afonso Ghizoni et al . Salomón Hakim: the man behind normal pressure hydrocephalus. Arq. Neuro-Psiquiatr. [Internet]. 2019 oct [cited 2021 Jan 03]. https://doi.org/10.1590/0004-282x20190096

8. Reeves BC, Karimy JK, Kundishora AJ, Mestre H, Cerci HM, Matouk C, Alper SL, Lundgaard I, Nedergaard M, Kahle KT. Glymphatic System Impairment in Alzheimer's Disease and Idiopathic Normal Pressure Hydrocephalus. Trends Mol Med. 2020 Mar;26(3):285-295. https://doi.org/10.1016/j.molmed.2019.11.008

9. Bae YJ, Choi BS, Kim J-M, Choi J-H, Cho SJ, Kim JH. Altered glymphatic system in idiopatic normal pressure hydrocephalus. Parkinsonism \& Related Disorders [Internet]. 2020 [citado 1 enero 2021];(82):56-60. Disponible en: https://doi.org/10.1016/ j.parkreldis.2020.11.0097. https://doi.org/10.1016/j.parkreldis.2020.11.009

10. Muñoz Suarez DR, Suarez Cadena FC, Ramos Vllegas Y, Padilla Zamora H, Moscote Salazar LR, Pacheco Hernández A. Hidrocefalia de Presión Normal. Rev. Ecuat. Neurol. [Internet] . 2017;25(1):40-44. 2020 [citado 1 enero 2021];26(3):285-292. https://doi.org/10.1016/j.molmed.2019.11.008

11. Saldarriaga-Cantillo A, Yepes-Gaviria V, Ricas JC. Normal. Presume hydrocephalus: Diagnostic delay. Biomédica. [Internet] 2020;40:656-63. https://doi.org/10.7705/biomedica.5382

12. Jameson J, Kasper D, Longo D, Fauci A, Hauser S, Loscalzo J. Harrison's Principles of Internal Medicine. 20. ${ }^{\mathrm{a}}$ ed. McGrawHill; 2018.

13. Herring W. Learning Radiology: Recognizing the Basics. 4. ${ }^{a}$ ed. España: Elsevier; 2020.

14. Ortega-Barnett J, Mohanty A, Desai S, Patterson J. Sabiston. Tratado de cirugía. 20. ${ }^{a}$ ed. España: Elsevier; 2018.

15. Sahuquillo Juan, Poc Maria. To Shunt or Not to Shunt Patients with Idiopathic Normal Pressure Hydrocephalus? A Reappraisal of and Old Question. Journal of Clinical Medicina. 2020. https://doi.org/10.3390/jcm9124120

16. Kazui H, Miyajima M, Mori E, Ishikawa M. Lumboperitoneal shut surgery for idiopatic normal pressure hydrocephalus: an open label randomised trial. Revista española de Geriatria y Gerontologia [Internet]. 2015 [citado 18 noviembre 2020];(14). http://dx.doi.org/10.1016/ S1474-4422(15)00046-0 
17. Ellenbogen, R., Sekhar, L., \& Kitchen, N. (2018). Principles of Neurological Surgery (4. ${ }^{a}$ ed.). España: Elsevier. España: Elsevier. 